
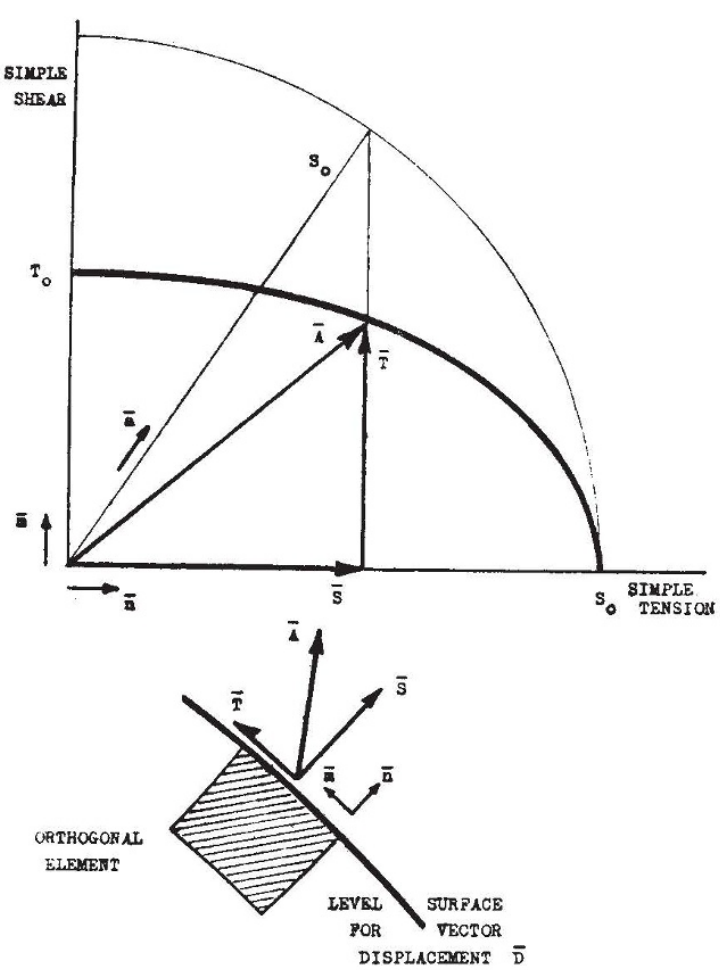

stress case. Such a linear form will allow easier analysis of many particular cases arising from the application of my theory of post-yield stress-strain ${ }^{5}$. In that theory the Huber criterion was used to give the simple tensile stress 'corresponding' to the complex stresses operating at any point in the yielded field. The new linear criterion can be used in the same way.

It is suggested tentatively that the criterion may apply to rupture, in which case $S_{0}$ is the simple tensile rupture stress for the metal. Examination of this hypothesis will require the analysis of stresses due to the usually large rupture deformations in some simple cases, and then comparison with the experimental values of the corresponding loads.

K. H. SWAINGER

Department of Civil Engineering,

Imperial College of Science and Technology, London, S.W.7.

March 8.

1 Timoshenko, S. P., "Strength of Materials", 2, 473-481 (Macmillan, 1941). Note eqn. 314 should read 0.577 and not 0.557 .

2 Nadai, A., "Plasticity", 59-69 (McGraw-Hill, 1931).

'Taylor, G. I., Phil. Trans. Roy. Soc., A, 323 (1932).

- Lode, W., Forschungsarbeiten a. d. Gebiete d. Ingineurwesens, No. 303 (VDI-Verlag, Berlin, 1928).

'Swainger, K. H., Phil. Mag., vii, 36, 443 (1945).

- Swainger, K. H., awaiting publication.

\section{Hiedemann Patterns at Very High Frequencies}

DIFFRaCTION patterns in liquids have recently been produced in this laboratory by using ultra-sonic waves of frequencies above $100 \mathrm{Mc}$./sec., and certain interesting features of such patterns have been studied $^{1}$. With a $2-\mathrm{mm}$. tourmaline plate, frequencies up to $103 \mathrm{Mc}$. $/ \mathrm{sec}$. are now communicated to a glass slab as sound waves and the corresponding diffraction patterns obtained by allowing light to pass through the glass slab, as was first done by Hiedemann ${ }^{2}$, but at much lower frequencies. The thickness of the glass slab used in our investigations is such that at these frequencies it oscillates at about its 300th harmonic.

Patterns due both to longitudinal and torsional sound waves are obtained either individually or simultaneously. It has been found that by attaching a suitable oscillator plate such as a $Y$-cut quartz to the glass slab, the longitudinal wave may be suppressed and the torsional wave made prominent and so on. The polarization characters of such diffraction patterns already reported by Hiedemann and others have all been verified. In an earlier communication referred to above, we have reported that the sound wave in water at such high frequencies causes diffraction, which appears to be very much liks reflexion in the Bragg sense. In these investigations, also, we have tried the effect of tilting the sound wave-front with reference to the incident light, and found that it introduces a marked asymmetry enhancing the intensity of the diffraction order on the appropriate side, but does not cause it to such an extent as altogether to extinguish the order on the opposite side at small inclinations, as has been found in water. This result is to be expected if we remember that the velocity of sound in glass is nearly four times as great as that in water, making the grating element at such high frequencies in the former four times as large as that in the latter. Consequently, effects due to tilting the sound wave-front obtained in glass at $100 \mathrm{Mc} / \mathrm{sec}$. should be like those obtained in water at $25 \mathrm{Mc}$./sec. only, which is indeed the case.

The velocities obtained for longitudinal waves of four different frequencies and a torsional wave $(46.46 \mathrm{Mc}$./sec.) in the glass slab are given below :

$\begin{array}{lrrrrr}\text { Frequency, in mc./sec. } & 13.24 & 20.74 & 47.04 & 103.55 & 46.46(T) \\ \text { Velocity, in } \mathrm{km} . / \mathrm{sec} . & 6.01 & 6.02 & 6.02 & 6.04 & 3.68\end{array}$

Elastic moduli calculated from the above data are $Y=8.03 \times 10^{11}$ dynes per sq. cm., $n=3.34 \times 10^{11}$ dynes per sq. em., and $\sigma=0 \cdot 201$.

\section{S. Bhagavantam \\ B. RamachandRa RaO}

Department of Physics,

Andhra University,

Waltair, India.

Feb. 28.

' [Nature, 159, 267 (1947). ]

${ }^{2}$ Naturwiss., 23, 705 (1935).

\section{Heat of Atomization of Carbon}

Is connexion with the series of communications under the above title $e^{1}$, I wish to direct attention to an old paper which contains an accurate computation of the metastable ${ }^{5} S$ state of the carbon atom ${ }^{2}$. The value given in that paper is $4 \cdot 3 \mathrm{eV}$. above the groundstate of carbon, and the uncertainty is less than $0.1 \mathrm{eV}$. This is equivalent to $100 \pm 2 \mathrm{kcal}$. $/$ mole.

The method of computation used in that paper is based on quantum mechanical perturbation theory. It differs radically from the usual iso-electronic extrapolation method, because it uses relations between energy states belonging to the same atom and its ions instead of comparing neighbouring atoms. The method gives surprisingly accurate predictions, especially for low levels of high multiplicity. For example, for the similar $s p^{3}{ }^{5} S$ levels in N II and O III, the computation yields 135 and $173 \mathrm{kcal} . / \mathrm{mole}$, whereas the observed values are 133 and 171 kcal./mole. 